\title{
Simulations on the Mixing Processes of Electrons and Positrons in a Magnetic Mirror Trap
}

\author{
Chikato KAGA \\ Graduate School of Advanced Sciences of Matter, Hiroshima University, \\ 1-3-1 Kagamiyama, Higashi-Hiroshima, Hiroshima 739-8530, Japan
}

(Received 20 October 2016 / Accepted 1 December 2016)

\begin{abstract}
Three-dimensional particle-in-cell simulations are performed to investigate the mixing process of low energy electrons and positrons for the confinement in a simple magnetic mirror with and without the plug potentials. According to the results of simulations, it is suitable to confine low energy electron-positron plasmas in a compact magnetic mirror trap.
\end{abstract}

(c) 2017 The Japan Society of Plasma Science and Nuclear Fusion Research

Keywords: electron-positron plasma, magnetic mirror, non-neutral plasma, 3D particle-in-cell

DOI: $10.1585 /$ pfr.12.1401001

\section{Introduction}

An electron-positron (e-p) plasma is an example of pair plasmas composed of the oppositely charged particles with equal masses. Relativistic e-p plasmas have been investigated in astrophysical objects such as pulsars [1,2], neutron star magnetospheres [3], galactic nuclei [4], jet plasmas [5], accretion disks [6], and many others [7, 8]. Also, laboratory experiments for e-p plasmas have been pursued. It was planed to confine relativistic e-p plasmas in a magnetic mirror [9]. High energy positrons emitted from radio isotopes (RI) [10] and moderated positrons [11] were confined in a magnetic mirror. Low energy e-p plasmas have been also studied theoretically [12-14] and some experimental studies have been performed. Low energy positrons from RI source were moderated [15] and accumulated in a Penning-Malmberg trap as a non-neutral plasma [16], and an electron-positron beam-plasma interaction was observed [17]. However, the simultaneous confinement of low energy electrons and positrons has not been realized yet.

Currently, there are projects to confine low energy ep plasmas in a magnetic dipole field [18] and a stellarator [19] with the use of low energy positrons from a nuclear reactor [20]. Also, the simultaneous confinement of low energy electrons and positrons with a compact magnetic mirror trap has been planned [21]. Although the confinement time in a magnetic mirror is short, it is easier to inject and accumulate a large number of electrons and positrons with the use of electrostatic potentials. Since the magnetic configuration is much simpler, plasma oscillation measurement becomes easier too. Here, three-dimensional particle-in-cell (3D PIC) simulations are performed to investigate the feasibility of mixing electrons and positrons in a compact magnetic mirror trap.

author'se-mail:d146073@hiroshima-u.ac.jp

\section{PIC Simulations}

A 3D PIC code Warp has been used to study various phenomena in non-neutral plasmas and beam dynamics. For example, space potentials of non-neutral electron plasmas in a magnetic field gradient were obtained previously [22]. In case of particle simulations, it is easy to understand charged particle distributions in phase space, space potential distributions, particle trajectories, and so on, which are much more difficult to obtain in experiments.

In the following simulations, the experimental setup for the simultaneous confinement of electrons and positrons in a compact magnetic mirror trap is reproduced [21]. The trap consists of four solenoid coils to create a magnetic mirror field and 14 electrodes to create various potentials inside the vacuum chamber. The schematic is shown in Fig. 1 (a). Two pair coils generate the magnetic mirror field with the mirror ratio $R \sim 5.88$. The calculated magnetic field strength on the axis of symmetry is plotted in Fig. 1 (b). The grounded vacuum chamber has the inner diameters of $200 \mathrm{~mm}$ at the center and $72 \mathrm{~mm}$ at both sides as shown in Fig. 1 (a). The electrodes named U1-U5 and D1-D5 have the inner diameter of $80 \mathrm{~mm}$ and axial length of $14 \mathrm{~mm}$ except the most inner electrodes (U1, D1) which have axial length of $25 \mathrm{~mm}$. The electrodes named U6, U7, D6, and D7 have the inner diameter of $56 \mathrm{~mm}$ and axial length of $90 \mathrm{~mm}$. These electrodes are used to create plug potentials outside the magnetic mirror field.

At first, an electron plasma and a positron plasma near equilibrium are prepared in harmonic potentials at $z \sim \pm 100 \mathrm{~mm}$ inside the magnetic mirror. The solid line in Fig. 1 (c) is the initial potential on the axis of symmetry for the separate confinement of an electron plasma and a positron plasma with the plug potentials. Both plasmas prepared in harmonic potential have $2 \times 10^{7}$ particles, in which $10^{4}$ macro particles with the weight of 2000 are used respectively. The number of particles can be increased with 
the larger weights and deeper harmonic potentials. The time step is $5.0 \times 10^{-11} \mathrm{~s}$ to resolve the cyclotron motion at the magnetic field of $0.13 \mathrm{~T}$. The grid sizes are $3.16 \mathrm{~mm}$ in $x, y$ and $9.14 \mathrm{~mm}$ in $z$. The initial velocity distribution is a gaussian profiles with the energy of $1 \mathrm{eV}$ perpendicular to $z$ and $2 \mathrm{eV}$ along $z$. A uniform circular distribution with the radius of $20 \mathrm{~mm}$ in $x, y$ and the gaussian distribution $(50 \mathrm{~mm})$ in axial direction $z$ are used. The Debye length is about $13 \mathrm{~mm}$. After the relaxation time of $10 \mu \mathrm{s}$, both plasmas have the root mean square (RMS) radius of $16 \mathrm{~mm}$ with a bell shape profile, the RMS length of $50 \mathrm{~mm}$, and the temperature less than $2 \mathrm{eV}$. Although the collisions between charged particles are not included in PIC simulations, numerical collisions lead to a quasi-equilibrium state. This distribution is used for both magnetic mirror confinement with and without the plug potentials.

Then, the mixing process starts from the simulation time $t=0 \mu \mathrm{s}$. In case of magnetic mirror confinement, the potentials on all the electrodes are grounded linearly in $1 \mu \mathrm{s}$ from $t=0.05 \mu \mathrm{s}$ to $1.05 \mu \mathrm{s}$ as shown by red and

(a)
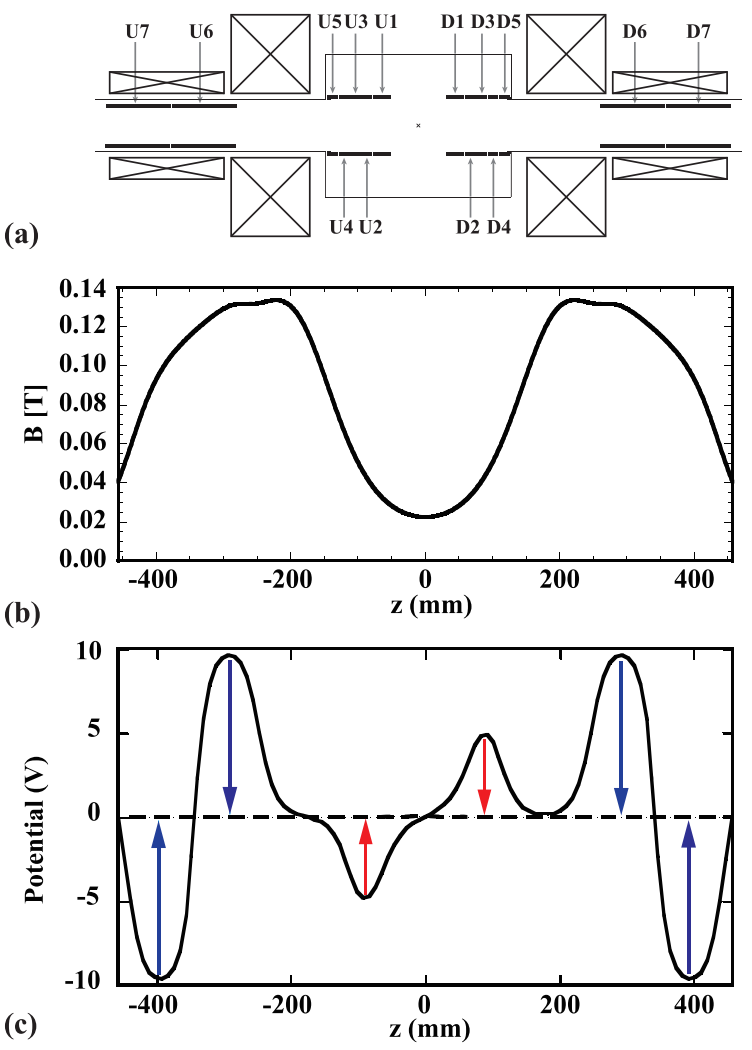

Fig. 1 (a) The configuration of the solenoid coils and electrodes. (b) The magnetic field strength on the axis of symmetry. (c) The solid line denote the initial potential profile on the axis of symmetry for preparing an electron plasma and a positron plasma. For the magnetic mirror confinement, all the electrodes are grounded (red and blue arrows) to make the flat potential denoted by the dashed line. For the plugging confinement, only the inner electrodes are grounded (red arrows). blue arrows in Fig. 1 (c). When electrons and positrons are confined with the plug potentials, only the harmonic potentials are grounded (red arrows). Similar calculations are performed with different weights of macro particles for electrons and positrons. The calculation continues until $t=5 \mu \mathrm{s}$, which takes about five days. Therefore, it is not feasible to estimate $E \times B$ drift and grad $B$ drift with current simulations, which have long time scales of more than several tens of micro seconds.

\section{Results and Discussions}

Presented here are two simulation examples. One is the simple magnetic mirror confinement without the plug potentials and the other is the magnetic mirror confinement with the plug potentials. Figure 2 (a1) to (a3) are the change of the potential on the axis of symmetry with the space charge of plasmas. Shown in Fig. 2 (b1) to (b3) are the macro particle distributions projected onto $z-x$ plane and shown in Fig. 2 (c1) to (c3) are those projected onto $z-v_{z}$ phase space in case of the magnetic mirror confinement, where the vertical axes $\left(v_{z} / c\right)$ are normalized by the speed of light $c$. Thus, $0.005 c$ corresponds to $\sim 6.4 \mathrm{eV}$ and $0.002 c$ corresponds to $\sim 1.0 \mathrm{eV}$. Figure $2(\mathrm{~d} 1)$ to $(\mathrm{d} 3)$ are
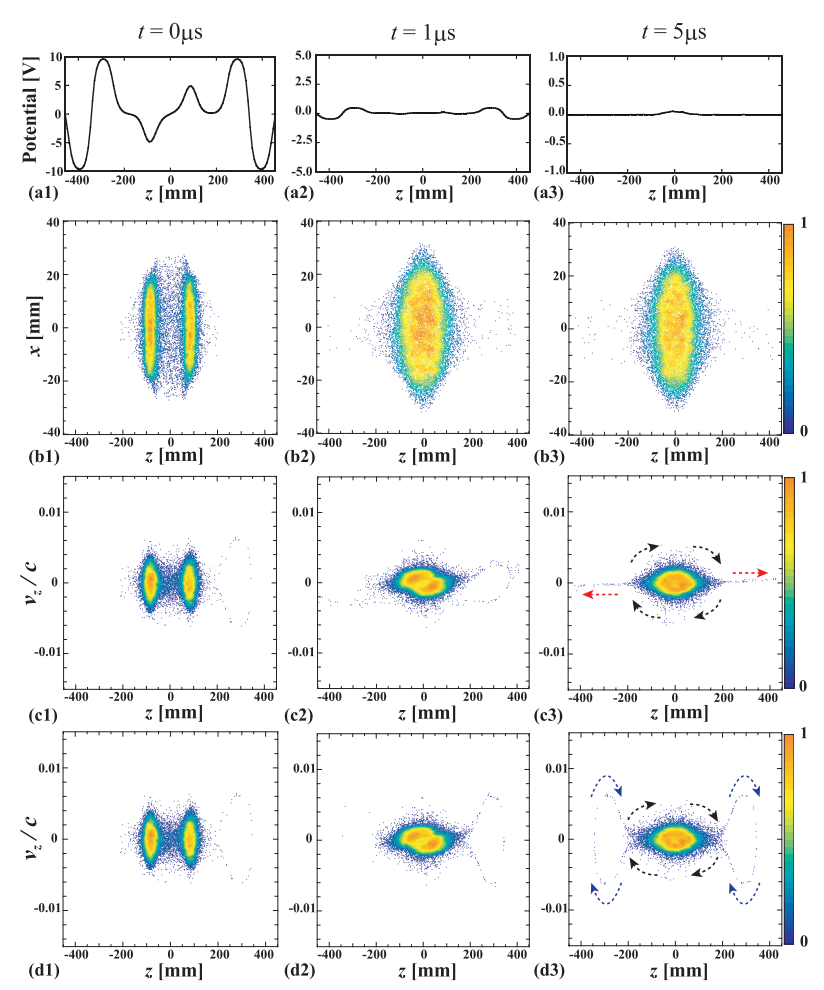

Fig. 2 (a1)-(a3) The potentials on the axis of symmetry. (b1)(b3) The macro particle distributions on $z-x$ plane. (c1)(c3) The macro particle distributions projected on $z-v_{z}$ plane without plug potentials. (d1)-(d3) The macro particle distributions projected on $z-v_{z}$ plane with the plug potentials. The numbers corresponds to the confinement time $t=0,1$, and $5 \mu \mathrm{s}$. 

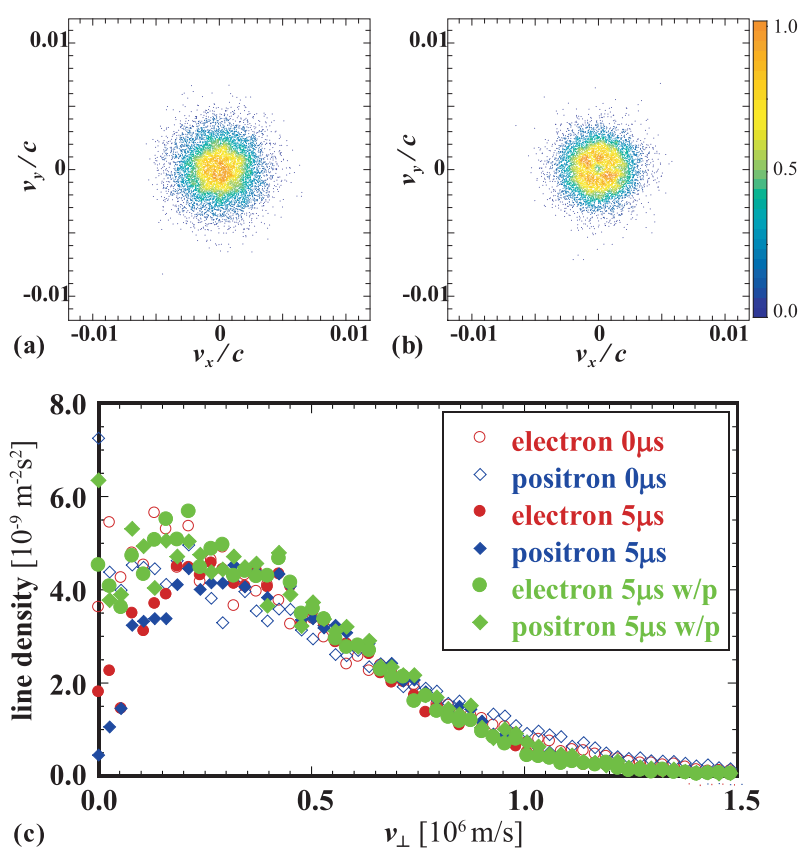

Fig. 3 The macro particle distributions projected on $v_{x}-v_{y}$ plane for the simple magnetic mirror confinement. (a) $t=0 \mu \mathrm{s}$ (b) $t=5 \mu \mathrm{s}$. (c) The line density profiles as a function of $v_{\perp}$. The red open circle is for electrons at $0 \mu \mathrm{s}$. The blue open diamond is for positrons at $0 \mu$ s. The solid red circle and blue diamond are for electrons and positrons at $5 \mu \mathrm{s}$ with the simple magnetic mirror confinement. The green circle and diamond are for electrons and positrons at $5 \mu \mathrm{s}$ with the plug potentials.

the $z-v_{z}$ phase space distributions with the plug potentials. The calibration bars are normalized by the maximum density in each figure. The numberings of figures correspond to the simulation time of $t=0,1$, and $5 \mu \mathrm{s}$.

At $t=0 \mu \mathrm{s}$, an electron plasma and a positron plasma are confined in the harmonic potentials in Fig. 2 (a1). As the potentials on electrodes approach to ground at $t=1 \mu \mathrm{s}$ (Fig. 2 (a2)), both plasmas expand inside the magnetic mirror as shown in Fig. 2 (b2). During the expansion, charged particles rotate clockwise direction in the phase space $\left(z-v_{z}\right)$ like a black dashed arrows in Figs. 2 (c3) and (d3). In case of the magnetic mirror confinement, particles inside the loss cones are lost along the magnetic field as shown by red dashed arrows in Fig. 2(c3). With the plug potentials, a small amount of electrons and positrons inside the loss cones are also confined. Blue dashed arrows in Fig. 2 (d3) represent the trajectories of electrons bouncing between the plug potentials. It is clear that the plug potentials prevent the particles in the loss cones from escaping from the trap. It is seen in Fig. 2 (a3) that space potential becomes less than $0.05 \mathrm{~V}$ for mixing electrons and positrons, which becomes more than $0.3 \mathrm{~V}$ without positrons.

Figures 3 (a) and (b) show the macro particle distributions of electrons and positrons projected on the $v_{x}-v_{y}$ plane at $t=0$ and $5 \mu$ s when they are confined in the simple
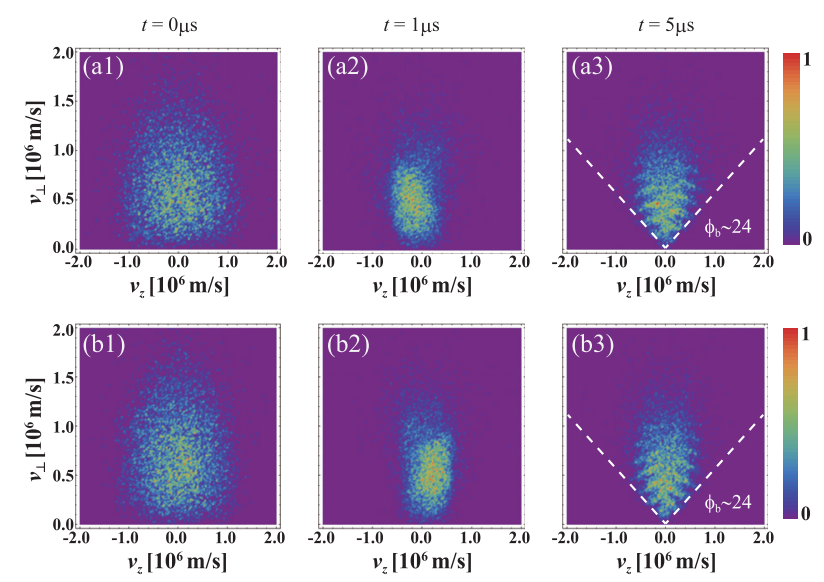

$v_{z}\left[10^{6} \mathrm{~m} / \mathrm{s}\right]$

$v_{z}\left[10^{6} \mathrm{~m} / \mathrm{s}\right]$

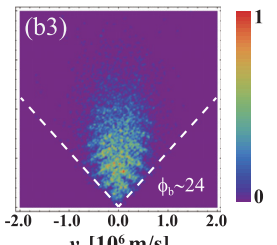

Fig. 4 (a1)-(a3) The macro particle distributions of electrons projected on $v_{z}-v_{\perp}$ plane for the simple magnetic mirror confinement. (b1)-(b3) The macro particle distributions of positrons projected on $v_{z}-v_{\perp}$ plane. The white dashed lines are loss cone boundaries calculated from the mirror ratio $R \sim 5.88$. The figure number correspond to $t=0,1$, and $5 \mu \mathrm{s}$.

magnetic mirror. The color scale represents the normalized area density of the macro particles in the $v_{x}-v_{y}$ plane. It is seen that the initial gaussian distribution at $t=0 \mu \mathrm{s}$ in Fig. 3 (a) becomes the hollow distribution at $t=5 \mu$ s when charged particles are confined in the simple magnetic mirror. This means that the charged particles inside the loss cones which are originally confined with the harmonic potentials are lost in the simple magnetic mirror. A similar velocity distribution can be observed due to the space potential of $\sim 0.3 \mathrm{~V}$ when only electrons are confined in a magnetic mirror.

Plotted in Fig. 3 (c) are the line density of the macro particle distributions as a function of $v_{\perp} \equiv \sqrt{v_{x}^{2}+v_{y}^{2}}$. The initial distributions in Fig. 3 (a) for electrons and positrons at $t=0 \mu$ s are denoted by open red circle and blue diamond, which are used for both magnetic mirror confinements with and without the plug potentials. The solid red circle and blue diamond are for electrons and positrons at $t=5 \mu$ s with the simple magnetic mirror confinement (Fig. 3 (b)). The green circle and diamond are for electrons and positrons at $t=5 \mu \mathrm{s}$ with the plug potentials. With the plug potentials, the line density profile of $v_{\perp}$ at $t=5 \mu \mathrm{s}$ remains similar to the initial one at $t=0 \mu$ s. On the other hand, it is clearly seen that the line density profile of the simple magnetic mirror confinement at $t=5 \mu$ s has the lower density near the $v_{\perp}=0$. These results reflect that the plug potential can suppress particles escaping from a magnetic mirror trap.

Figure 4 shows the macro particle distributions projected on the $v_{z}-v_{\perp}$ plane for electrons and positrons normalized by the peak intensity of each figure. The figure numbers correspond to $t=0,1$, and $5 \mu$ s. The macro particle distributions of electrons projected on $v_{z}-v_{\perp}$ plane for 

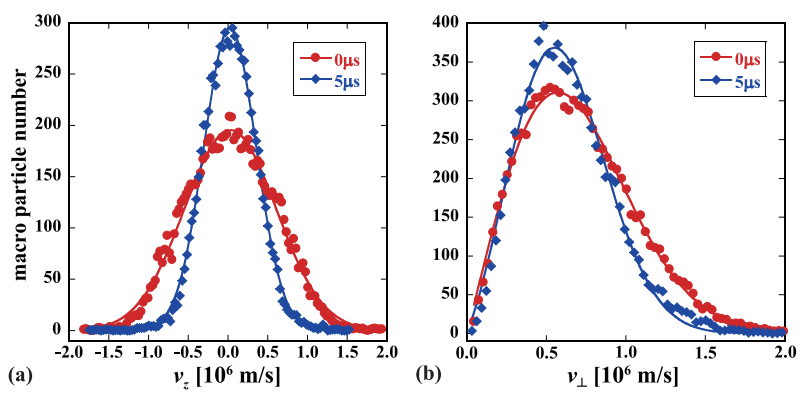

Fig. 5 The velocity distribution for electrons with the simple magnetic mirror confinement at $t=0$ (red circles) and $5 \mu$ s (blue diamonds). Solid lines show the fitting lines with Maxwell distribution. (a) The velocity distribution in $z$ direction. (b) The velocity distribution perpendicular to the magnetic field.

the simple magnetic mirror confinement are in Figs. 4 (a1)(a3) and those for positrons are in Figs. 4 (b1)-(b3). White dashed lines in Figs. 4 (a3) and (b3) represent the loss cone boundaries in velocity space, which has the loss cone angle of $\phi_{b} \sim 24^{\circ}$ corresponding to the mirror ratio $R \sim 5.88$.

At the beginning, an electron plasma and a positron plasma are confined by harmonic potentials inside the magnetic mirror, where velocity distributions become as seen in Figs. 4 (a1) and (b1). As the harmonic potentials are grounded at $1.05 \mu \mathrm{s}$, both plasmas expand toward the center of the trap. Thus, the macro particle distribution in the $v_{z}-v_{\perp}$ plane for electrons shifts $v_{z} \leq 0$ and the one for positrons shifts $v_{z} \geq 0$, respectively, as shown in Figs. 4(a2) and (b2). Since the change of the harmonic potentials to the ground is adiabatic process, the adiabatic bounce invariant $J_{\|}=\oint v_{z} d z$ is preserved [23]. Therefore, the distribution in $v_{z}$ direction shrinks as shown in Fig. 5 (a). Figure 5 (a) shows the velocity distribution in $z$ direction for electrons with the simple magnetic mirror confinement at $t=0 \mu \mathrm{s}$ and $t=5 \mu \mathrm{s}$, which correspond to red circles and blue diamonds. Figure 5 (b) shows velocity distribution in perpendicular direction. The temperature of electrons and positrons in $z$ direction at $t=0 \mu \mathrm{s}$ is about $1.8 \mathrm{eV}$ on average, which become about $0.6 \mathrm{eV}$ at $t=5 \mu \mathrm{s}$. The macro particle distribution in $x, y$ plane also expands in the radial direction along the magnetic field. So, the temperature of electrons and positrons perpendicular to the magnetic field at $t=0 \mu \mathrm{s}$ is about $0.8 \mathrm{eV}$ on average and those at $t=5 \mu \mathrm{s}$ is about $0.5 \mathrm{eV}$. Similar decreases of temperatures during the adiabatic potential change are also reproduced only with electrons. As a result, most of the particles remain outside loss cones. The calculated survival ratio of the charged particles is about $95 \%$ for simple magnetic mirror confinement. This is larger than $\sim 91 \%$, which is the original particles outside loss cones at $t=0 \mu \mathrm{s}$ calculated for $R \sim 5.88$. Therefore, the adiabatic potential change enhances the confinement of charged particles inside the magnetic mirror.
As the particles move to the center of the trap when the potential barriers are grounded, the macro particle distributions start oscillation in the $v_{z}-v_{\perp}$ plane. It is observed in Figs. 4 (a3) and (b3) that particles of the plasma confined in the magnetic mirror oscillate between white dashed lines in $v_{z}-v_{\perp}$ plane. It means that charged particles move on the concentric ellipse due to energy conservation and its oscillation period depends on the magnetic moment $\mu$. The oscillation frequency becomes $f=\omega_{0} / 4 K(k)$, where $\omega_{0} \equiv \frac{\pi}{L} v \sin \phi_{0} \sqrt{R-1}$ and $k \equiv \cot \phi_{0} / \sqrt{R-1}$. Here, The function $K(k)$ is the complete elliptic integral of the first kind, and $L$ is the axial distance between the maximum points of magnetic field. The phase $\phi_{0}$ is the pitch angle at $z=0$ and $v \sin \phi_{0}=\sqrt{v_{z}^{2}+v_{\perp}^{2}} \sin \phi_{0}$ is the $v_{\perp}$ at $z=0$. The striped pattern in Figs. 4 (a3) and (b3) can be explained qualitatively by single particle oscillations. Since the oscillation frequency is proportional to $v$ for the fixed $\phi_{0}$, the particles with higher energy oscillate much more. The macro particle distributions in the velocity space with the plug potentials are almost the same with those for the simple magnetic mirror confinement.

\section{Conclusion}

The mixing processes of electrons and positrons in a magnetic mirror trap with and without the plug potentials were evaluated with 3D PIC simulation Warp.

Under the condition that collisions between charged particles and back ground neutrals can be ignored, it was confirmed that a compact magnetic mirror trap with harmonic potentials inside a magnetic mirror can be used to confine low energy e-p plasmas. To consider effects of $E \times B$ and $\operatorname{grad} B$ drifts, improved simulations are necessary to calculate a longer time scale.

Preparing electron and positron plasmas in harmonic potentials enhances the confinement of e-p plasma through the adiabatic potential changes. Since the collision frequency between charged particles in the present simulation parameters is expected to be $23 \mathrm{~Hz}$, it is thought that the e-p plasma oscillation in the range of a few $\mathrm{MHz}$ can be observed.

\section{Acknowledgments}

The author is grateful to Dr. H. Higaki, Dr. K. Ito, and Dr. H. Okamoto for valuable discussion and suggestion. The author also wish to thank Dr. K. Osaki and Dr. K. Fukushima for technical support for the simulations.

[1] P. Goldreich and W.H. Julian, Astrophys. J. 157, 869 (1969).

[2] F.C. Michel, Rev. Mod. Phys. 54, 1 (1982).

[3] C. Max and F.W. Perkins, Phys. Rev. Lett. 29, 1731 (1972).

[4] M.C. Begelman, R.D. Blandford and M.D. Rees, Rev. Mod. Phys. 56, 255 (1984).

[5] J.F.C. Wardle, D.C. Homan, R. Ojha and D.H. Roberts, Nature 395, 457 (1998). 
[6] J.R. Orsoz, R.A. Remillard, C.D. Bailyn and J.E. McClintock, Astrophys. J. 478, L83 (1997).

[7] J. Daniel and T. Tajima, Astrophys. J. 498, 296 (1998).

[8] T. Piran, Rev. Mod. Phys. 76, 1143 (2004).

[9] V. Tsytovich and C.B. Wharton, Comments Plasma Phys. Control. Fusion 4, 91 (1978).

[10] G. Gibson, W.C. Jordan and E.J. Lauer, Phys. Fluids 6, 116 (1963).

[11] H. Boehmer, M. Adams and N. Rynn, Phys. Plasmas 2, 4369 (1995).

[12] N. Iwamoto, Phys. Rev. E 47, 604 (1993).

[13] G.P. Zank and R.G. Greaves, Phys. Rev. E 51, 6079 (1995).

[14] M.W. Verdon and D.B. Melrose, Phys. Rev. E 77, 046403 (2008).

[15] P.J. Schultz and K.G. Lynn, Rev. Mod. Phys. 60, 701 (1988).

[16] C.M. Surko, M. Leventhal and A. Passner, Phys. Rev. Lett.
62, 901 (1989).

[17] R.G. Greaves and C.M. Surko, Phys. Rev. Lett. 75, 3846 (1995).

[18] H. Saitoh, J. Stanja, E.V. Stenson, U. Hengenhahn, H. Niemann, T. Sunn Pedersen, M.R. Stoneking, C. Piochacz and C. Hungenschmidt, New J. Phys. 17, 103038 (2015).

[19] T. Sunn Pedersen, J.R. Danielson, C. Hungenschmidt, G. Marx, X. Sarasola, F. Schauer, L. Schweikhard and C.M. Surko, New J. Phys. 14, 035010 (2012).

[20] C. Hugenschmidt, C. Piochacz, M. Reiner and K. Schreckenbach, New J. Phys. 14, 055027 (2012).

[21] H. Higaki, S. Sakurai, K. Ito and H. Okamoto, Appl. Phys. Express 5, 106001 (2012).

[22] H. Higaki, K. Fukata, K. Ito, H. Okamoto and K. Gomberoff, Phys. Rev. E 81, 016401 (2010).

[23] A.W. Hyatt, C.F. Driscoll and J.H. Malmberg, Phys. Rev. Lett. 59, 2975 (1987). 